\title{
Application of Improved Double Ant Colony Algorithm in The Robot Path Planning
}

\author{
Ge Bin ${ }^{1, a}$, Houyuan Sheng ${ }^{2, b}$ \\ ${ }^{1}$ Dalian University, Dalian 116622, China \\ ${ }^{2}$ Dalian University, Dalian 116622, China \\ agebin58@163.com, bshenghouyuan@163.com
}

Keywords: double ant colony algorithm, mobile robot, path planning, combined optimization.

\begin{abstract}
Traditional ant colony algorithm is applied to mobile robot path planning, existing to slowly solve, slowly convergence speed, easy to fall into local optimal .A kind of double ant colony optimization based on combined optimization is proposed. Forward ant searched path be combined with backward ant searched path when two groups of ants in current generation completed a path search. Then the new paths were sorted, selecting the better path for global pheromone updating, combining the thought of MAX-MIN ant colony algorithm. Simulation experiment shows the improved ant colony algorithm can well solve the problems of the traditional ant colony.
\end{abstract}

\section{Introduction}

The planning of mobile robots is that the robot in an obstructed environment search an optimal path according to one or more rules (such as minimum energy consumption, the shortest path).The optimal obstacle-avoidance path is from the starting point to goal point. Ant colony algorithm has many advantages like information positive feedback effect, excellent parallel computing, strong ability of heuristic searching, etc. But ant colony algorithm has many disadvantages like lack of initial pheromone, solving slowly, easily falling into local optimal solution. Double ant colony optimization based on combined optimization is proposed.

\section{The combination optimization of path}

When two groups of ants in current generation completed a path search, forward ant searched path be combined optimize with reverse ant searched path to combine into the better paths. Forward ant searched path is defined as $P 1$. Reverse ant searched path is defined as $P 2$. Combined path is defined as $P$. The combination optimization can be divided into three categories.

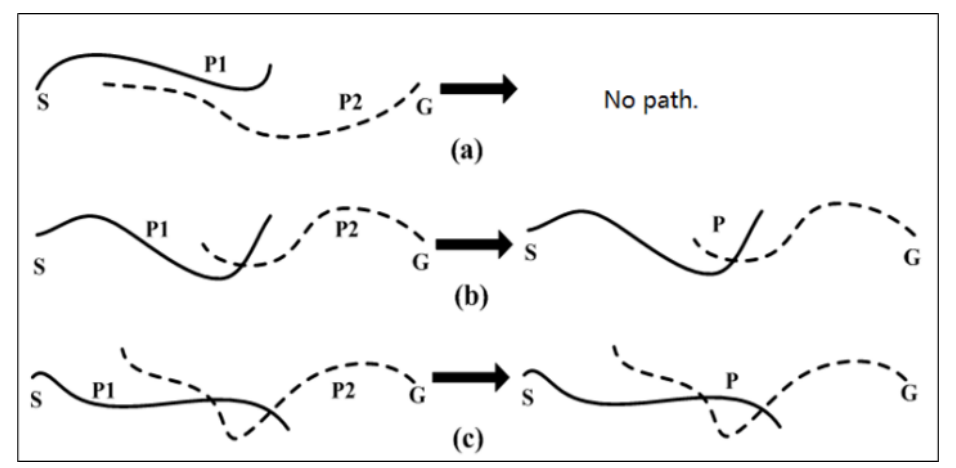

Fig. 1 Two paths are not complete 


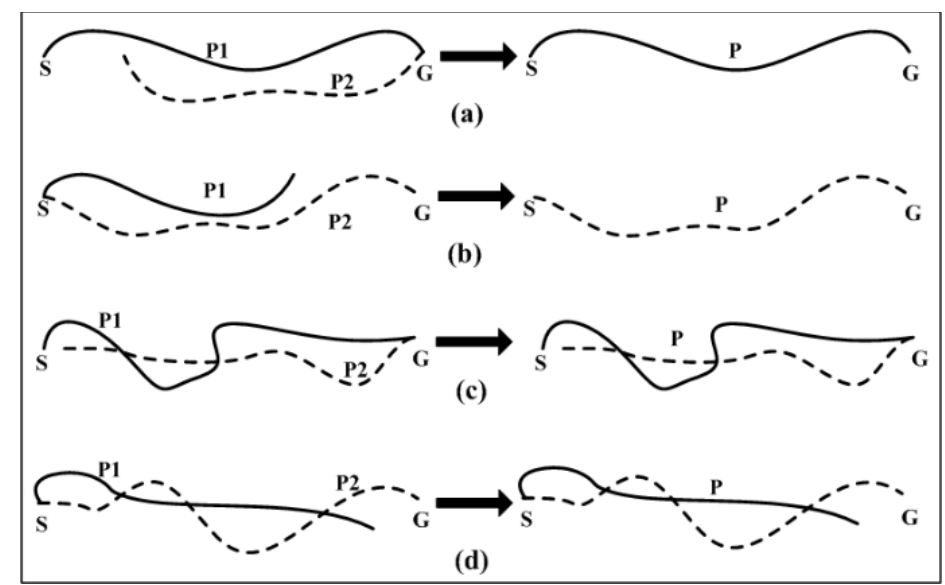

Fig. 2 Only a complete path

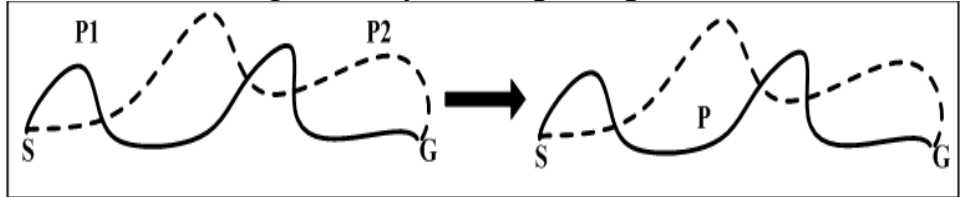

Fig .3 Two paths are complete

As shown in the Fig. 1, two incomplete path can also be combined into a complete path, improving the ability of ant colony algorithm plan path. As shown in the Fig. 3, the incomplete path can optimize the complete path, increasing the diversity of paths. Combination optimization enhance the information interaction between the two groups of ants, accelerating the speed of solution, increasing the diversity of solution.

\section{Enhanced Ant Colony Optimization Algorithm}

According to the above definitions, the procedure of enhancement ant colony algorithm is implemented as follow.

Step 1: Load the image and preprocess the image.

Step 2: Initializing parameters of the ant colony algorithm used in the process of operation. Let $k=1, m=1$.

Step 3: If $k<=K$, that is not the number of iterations, go to Step 4. If $k>K$, the algorithm is over and go to Step 15.

Step 4: Place the ant $m$ on the starting point $S$. The current node number is assigned to $i$. Put point $S$ in the path node number matrix ROUTE. KXW is the reachable neighbor set, which in the neighborhood of grid $i$ and not on the current path and not belongs to the obstacle grids matrix $Z A D$.

" $C=$ setdiff $(A, B)$ " denotes that function returns the values in $A$ that are not in $B$ with no repetitions.

$$
K X W=\operatorname{setdiff}\left(L I,\left[Z A D^{\prime}, \text { ROUTE }\right]\right)
$$

Step 5: If $i \neq E$ and matrix $K X W$ is not empty, continue to look for the next path point $j$, otherwise go to Step 7. State transition probability, the ant $m$ choosing node $j$, is calculated by Eq.(2). $\alpha$ is the relative importance of pheromone. $\beta$ is the relative importance of distance heuristic function. Selecting the next path points by roulette wheel selection, then the node number is assigned to $j$.

$$
P_{i j}^{\mathrm{k}}=\frac{\tau_{i j}^{\alpha} * \eta_{i j}^{\beta}}{\sum_{s \in K X W} \tau_{i s}^{\alpha} * \eta_{i s}^{\beta}}
$$

Step 6: Add the node $j$ to the path matrix ROUTE. Let $i=j$. Find out that the next step can choose the reachable set $K X W$, go to Step 5 . 


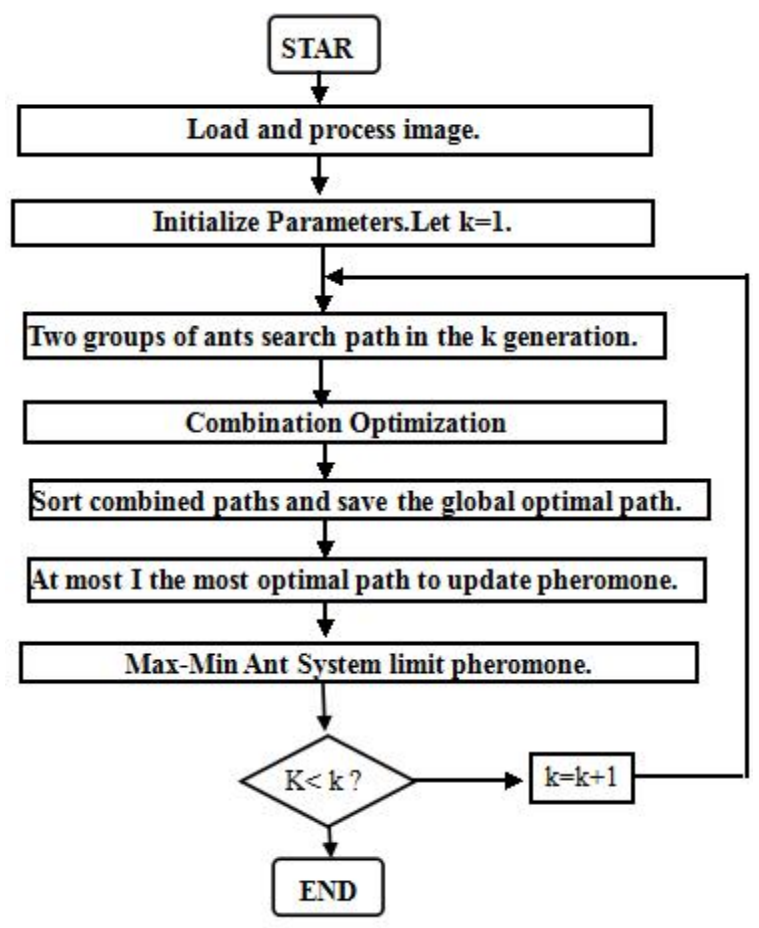

Fig. 4 The algorithm flow chart

Step 7: Save the path matrix ROUTE to unit array ROUTES $\{k, m\}$.Let $m=m+1$.If $m<=M / 2$, then go to Step 4. Otherwise go to Step 8.

Step 8: The backward ants search path according to Step 4-Step 7.

Step 9: Forward ant searched path be combined with backward ant searched path.

Step 10: Sort combined paths and update the global optimal path.

Step 11: The dissipation of pheromone is is calculated by Eq.(3).

$$
\left\{\begin{array}{l}
\tau_{\mathrm{ij}}(t)=\tau_{\min }, \quad \text { if } \quad \tau_{\mathrm{ij}}(t)<\tau_{\text {min }} \\
\tau_{\mathrm{ij}}(t)=(1-\rho) \tau_{\mathrm{ij}}(t-1), \quad \text { else }
\end{array}\right.
$$

Step 12: Choose at most $I$ the most optimal path is used to update global pheromone increment and the global optimal path needs additional compensation.

Step 13: Update global pheromone. Updated formula is as follows.

$$
\left\{\begin{array}{l}
\tau_{\mathrm{ij}}(t)=\tau_{\max }, \quad \text { if } \quad \tau_{\mathrm{ij}}(t)>\tau_{\max } \\
\left.\tau_{\mathrm{ij}}(t)=\tau_{\mathrm{ij}}(t)+\Delta \tau_{\mathrm{i}\}} \mathrm{t}\right) \quad \text { else }
\end{array}\right.
$$

Step 14: Let $k=k+1, m=1$. Go to Step 3 .

Step 15: Output the best path.

\section{The Simulation Test And Performance Analysis}

Under two complex environments, the algorithm (CCDACA) in literature [4] and the improved ant colony algorithm (IDACA) dose the contrast experiment. Fig. 5 and Fig. 6 show the simulation results respectively. 


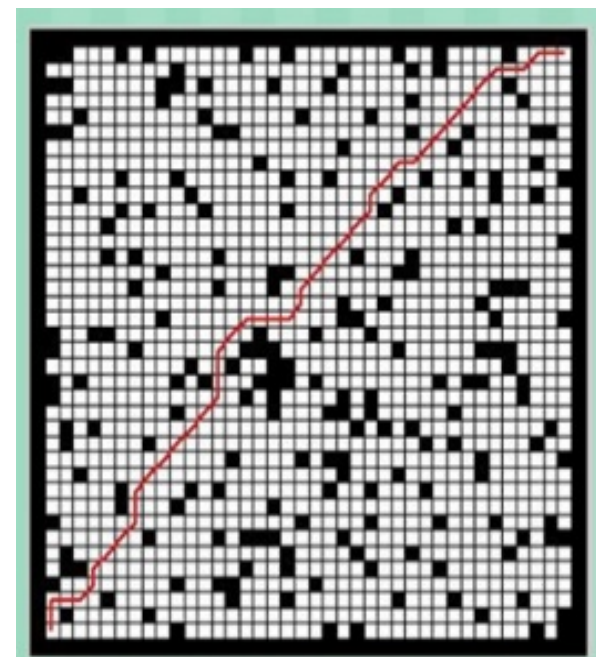

(a) CCDACA

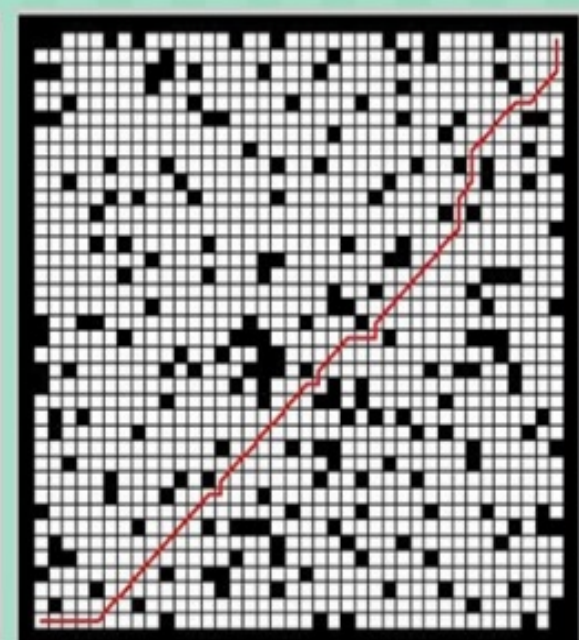

(b) IDACA

Fig. 5 Result under the environment a

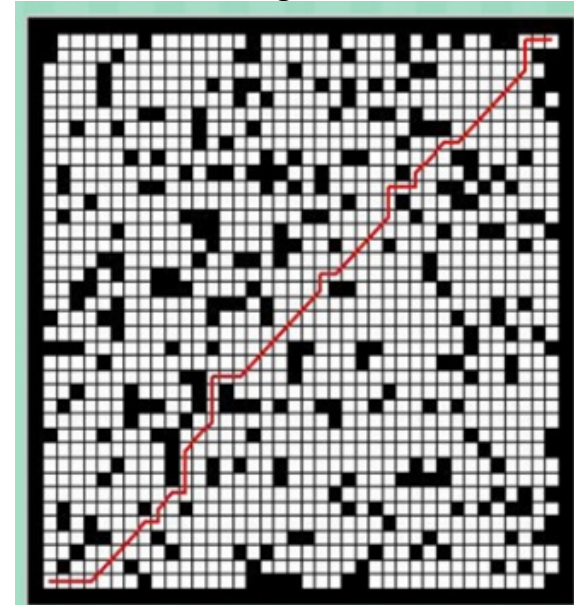

(a) CCDACA

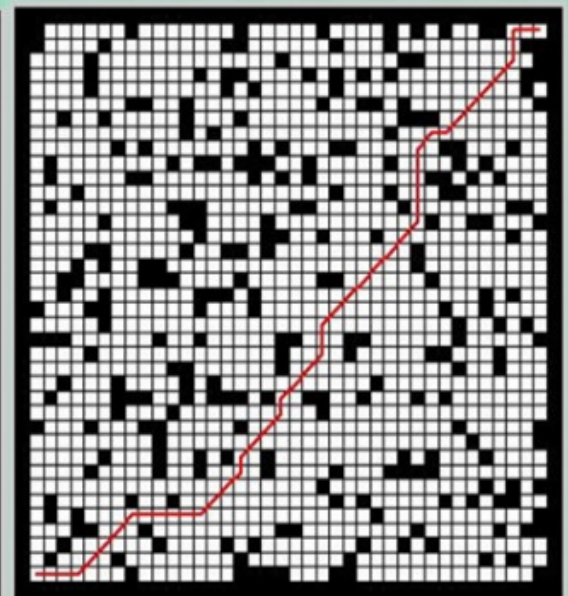

(b) IDACA

Fig. 6 Result under the environment $b$

To eliminate the effects of accidental factors, each algorithm does 20 simulation experiments in each environment. Record the length of the path optimal, the number of its knee and the time of path planning every time, then average its. The simulation result is as table 1.

Table 1 Two algorithms' performance comparison

\begin{tabular}{ccccc}
\hline Environment & Algorithm & Length & Knee & Time \\
\hline \multirow{2}{*}{$\mathrm{a}$} & CCDACA & 58.5976 & 19 & $8.203 \mathrm{~s}$ \\
& IDACA & 57.2345 & 17 & $6.302 \mathrm{~s}$ \\
\hline \multirow{2}{*}{$\mathrm{b}$} & CCDACA & 58.1834 & 21 & $9.804 \mathrm{~s}$ \\
& IDACA & 57.7692 & 15 & $7.080 \mathrm{~s}$
\end{tabular}

We can see from the above table that IDACA generates the shorter path than CCDACA, taking less time, having less knees. The improved algorithm is correct and effective.

\section{Summary}

Double ant colony algorithm strengthen the information interaction between the two groups of ants through combination optimization of the path. This accelerate the solving speed and increase the diversity of path. Sorting combined path, the better path and the global optimal path is used to update global pheromone, improving the convergence speed. The algorithm does not easily fall into local optimum, because Max-Min Ant System's idea is used. Simulation results show that the improved algorithm accelerate the solving speed and convergence speed. 


\section{References}

[1] WANG Xian, WANG Wei. Robot path planning based on ant colony optimization and particle swarm optimization. Computer Systems and Applications. 2011, 20(9): 98-102.

[2] ZHAO Juan-ping, GAO Xian-wen. Improved ant colony algorithm of path planning for mobile robot. Control Theory Applications. 2011, 28(4): 457-461.

[3] ZHU Qing-bao, MA Ping. A robot path planning algorithm based on scout ants in collaboration with foraging ants. Control and Decision. 2009, 24(4): 601-605.

[4] SUN Chun-zhe, LIN Ju-guang. An double ant colony algorithm in concave obstacle environment for global path planning. Transactions of the Chinese Society for Agricultural Machinery, 2008, 39(7): 149-153.

[5] LIU Xu-dong. Research and application on the hybrid colony algorithm. Anhui: Anhui University of Science and Technology, 2013. 